\title{
Spontaneous emergence of overgrown molar teeth in a colony of Prairie voles (Microtus ochrogaster)
}

\author{
Andrew H Jheon ${ }^{1}$, Michaela Prochazkova ${ }^{1,2}$, Michael Sherman ${ }^{3}$, Devanand S Manoli ${ }^{4,5}$, Nirao M Shah ${ }^{5}$, \\ Lawrence Carbone ${ }^{3}$ and Ophir Klein ${ }^{1}$
}

Continuously growing incisors are common to all rodents, which include the Microtus genus of voles. However, unlike many rodents, voles also possess continuously growing molars. Here, we report spontaneous molar defects in a population of Prairie voles (Microtus ochrogaster). We identified bilateral protuberances on the ventral surface of the mandible in several voles in our colony. In some cases, the protuberances broke through the cortical bone. The mandibular molars became exposed and infected, and the maxillary molars entered the cranial vault. Visualisation upon soft tissue removal and microcomputed tomography (microCT) analyses confirmed that the protuberances were caused by the overgrowth of the apical ends of the molar teeth. We speculate that the unrestricted growth of the molars was due to the misregulation of the molar dental stem cell niche. Further study of this molar phenotype may yield additional insight into stem cell regulation and the evolution and development of continuously growing teeth.

International Journal of Oral Science (2015) 7, 23-26; doi:10.1038/ijos.2014.75; published 30 January 2015

Keywords: continuously growing teeth; molar phenotype; mutation; stem cell regulation; voles

\section{INTRODUCTION}

All rodents are characterized by continuously growing incisors. Studies in mice have demonstrated that incisor renewal is supplied by stem cells that are housed in distinct epithelial and mesenchymal niches. The incisor epithelial niches are composed of the labial and lingual cervical loop (CL) regions that are retained in adult mice and regulate continuous growth. ${ }^{1-4}$ Dental mesenchymal stem cells reside in the areas between and adjacent to the CL regions ${ }^{5-6}$ and give rise to cells such as odontoblasts that form dentin, the mineralized tissue that underlies enamel. In contrast to mouse incisors, mouse molars are similar to all human teeth and do not grow continuously. However, mouse incisors and molars undergo similar developmental events at early stages. Notable differences occur during incisor development with the presence of a vestibular lamina, retention of the CL regions and the formation of a single, primary enamel knot, but no secondary enamel knots.

Prairie voles (Microtus ochrogaster), similar to mice, possess a reduced dentition that is composed of one incisor and three molar teeth (Figure $1 \mathrm{a}-1 \mathrm{~g}$ ) in each of the four quadrants. However, in contrast to mice (Figure $1 \mathrm{~h}$ and $1 \mathrm{i}$ ), voles possess continuously growing molars and incisors. In human teeth and mouse molars, the roots are generated through the formation the Hertwig's epithelial root sheath (HERS), which is derived from the inner enamel epithelia (IEE) and outer enamel epithelia (OEE) (Figure 1j). The development of the HERS is soon followed by the arrest of tooth growth. This leads to the presence of HERS remnants called the epithelial cell rests of
Malassez along the root surface. The continuously growing vole molar and the mouse incisor do not produce HERS or epithelial cell rests of Malassez, but maintain stellate reticulum (SR) cells housed between the IEE and OEE (Figure $1 \mathrm{~g}$ ). The SR and OEE regions of the vole molar and rodent incisor labial CL are presumed to house the stem cells that fuel continuous growth. ${ }^{7}$ However, relatively little is known about the molar stem cell niche ${ }^{7}$ compared to the incisor stem cell niche. ${ }^{1-4,8}$

Here, we present data from several Prairie voles (Microtus ochrogaster) in our colony presenting with dramatic overgrowth of the molar teeth, which was likely due to a spontaneous mutation leading to defects in the adult dental stem cell regulation. The inheritance profile of the molar phenotype suggested a multifactorial aetiology.

\section{METHODS}

Voles

Our animal research facility is registered with the US Department of Agriculture (USDA) and has had continuous Association for Assessment and Accreditation of Laboratory Animal Care (AAALAC) accreditation since 2004. All voles in this colony were derived from founder voles from the University of California at Davis originating from the colony maintained by the laboratory of Dr Karen Bales. All animals are managed according to Institutional Animal Care and Use Committee (IACUC) approved protocols that are consistent with all applicable regulations as prescribed in the USDA Animal Welfare Regulations ${ }^{9}$ and in accordance with the Guide for the Care and Use of Laboratory Animals. ${ }^{10}$

${ }^{1}$ Department of Orofacial Sciences and Program in Craniofacial and Mesenchymal Biology, University of California, San Francisco (UCSF), San Francisco, USA; ${ }^{2}$ Department of

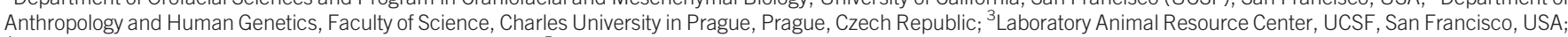
${ }^{4}$ Department of Psychiatry, UCSF, San Francisco, USA and ${ }^{5}$ Department of Anatomy, UCSF, San Francisco, USA

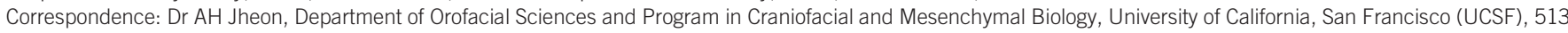
Parnassus Avenue S505, San Francisco CA 94143, USA

E-mail: andrew.jheon@ucsf.edu

Accepted 8 October 2014 

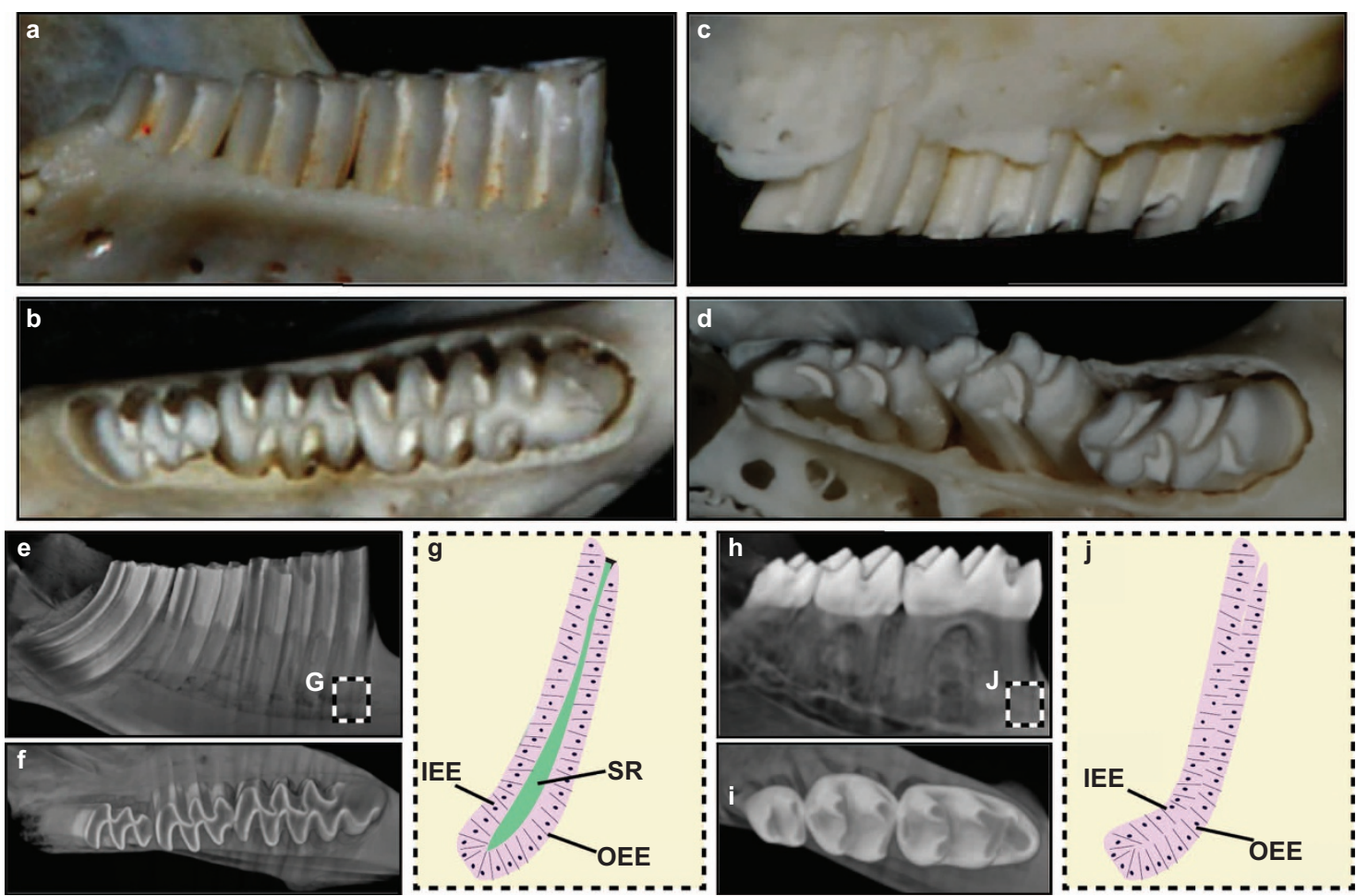

Figure 1 Vole and mouse teeth. (a, b) Mandibular vole molars in the lateral (a) and occlusal (b) views. (c, d) Maxillary vole molars in the lateral (c) and occlusal (d) views. (e, f) MicroCT images of vole molars in the lateral (e) and occlusal (f) views. (g) The apical region of the continuously growing vole molar is composed of the IEE, OEE and SR. (h, i) MicroCT images of mouse molars in the lateral (h) and occlusal (i) views. (j) The apical region of the rooted and non-continuously growing mouse molar is composed of the IEE and OEE and is devoid of the SR. Images are not to scale. IEE, inner enamel epithelium; mciroCT, microcomputed tomography; OEE, outer enamel epithelium; SR, stellate reticulum.

The voles were housed with standard rodent temperature and humidity (68-72\% and 30\%-70\% humidity) and lived in standard polycarbonate rat cages $(20 \mathrm{~cm} \times 40 \mathrm{~cm} \times 20 \mathrm{~cm})$. The voles received 5058 Breeder chow (LabDiet, St. Louis, MO, USA) in hoppers and 5826 Hi-Fiber Rabbit chow (LabDiet, St. Louis, MO, USA) on the floor of the cage. The bedding was purchased from Sanichips (P.J. Murphy Forest Products, Montville, NJ, USA), and the voles were maintained on a 14:10-h light cycle set for 7 a.m. to 9 p.m. (on) and 9 a.m. to 7 p.m. (off).

\section{Preparation of specimens and analyses}

Adult voles were decapitated, and the skin was removed from the heads. The heads were either fixed with $4 \%$ paraformaldehyde in phosphate buffer solution (PBS) for $48 \mathrm{~h}$ at $4{ }^{\circ} \mathrm{C}$ and then stored in $70 \%$ ethanol or the soft tissue was removed by dermestid beetles. Photos were obtained using a Nikon D3200 DSLR Camera. MicroCT analysis was performed with a MicroXCT-200 (Xradia, Pleasanton, CA, USA) through the MicroCT Imaging Facility at University of California, San Francisco (UCSF).

\section{RESULTS}

Three animals (one male, two females) in our vole colony presented with two bilateral protuberances on the ventral surface of the mandible (Figure 2). Since these initial animals were identified, we generated five additional animals (three males, two females) with similar phenotypes by breeding the three affected voles with wild-type voles from the same colony. The sizes of the bilateral protuberances varied from $4-8 \mathrm{~mm}$ in diameter and 4-6 $\mathrm{mm}$ in height, and the protuberances were not detected before 5 months of age. Overgrown upper
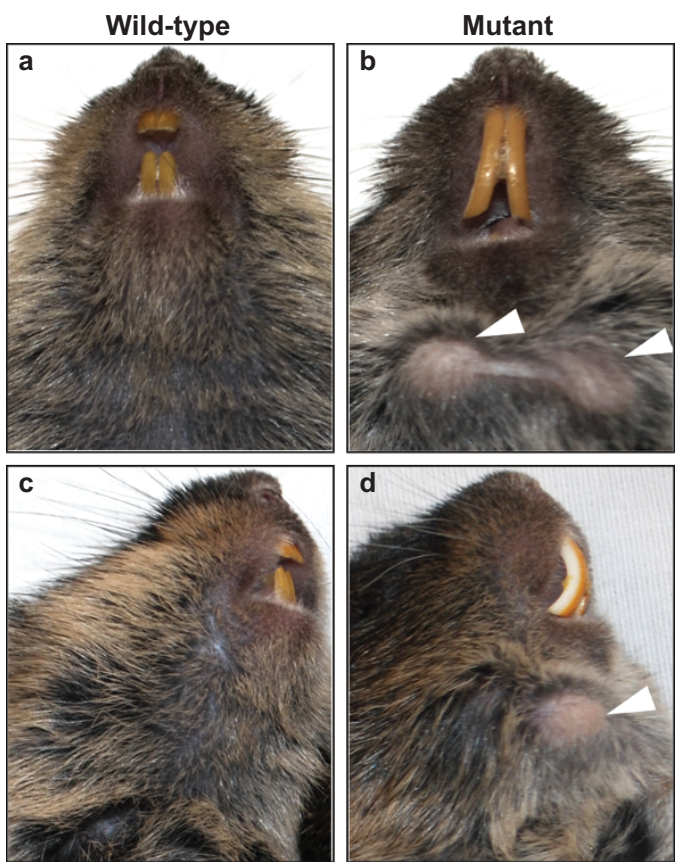

Figure 2 Wild-type and mutant voles. (a-d) Images of wild-type (a, c) and mutant (b, d) voles demonstrate the presence of bilateral protuberances on the ventral surface of the mandible in the mutant (white arrowheads). Note the elongated maxillary incisors in the mutant vole. 

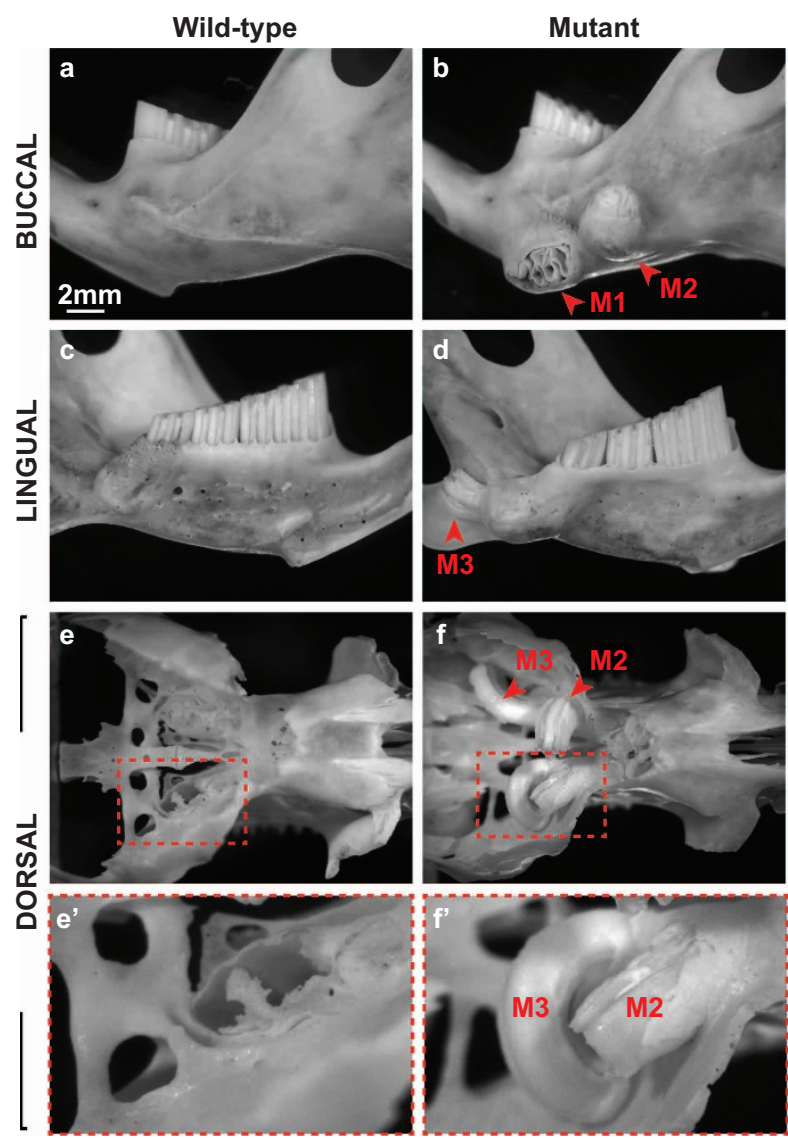

Figure 3 Skeletal analysis revealed the overgrowth of the apical region of mutant molars. (a-f') Images of wild-type (a, c, e, e $\mathbf{e}^{\prime}$ ) and mutant (b, d, f, $\mathbf{f}^{\prime}$ ) hemimandibles (a-d) and the cranial base (e- $\left.\mathbf{f}^{\prime}\right)$ in the buccal, lingual, and dorsal views demonstrate the uncontrolled growth of the three mutant molars (M1, M2, $M 3)$. Note the compromise in cortical bone due to the overgrowth of $M 1$ and $M 3$ in the mutant hemimandible and the breech in the cranial base and entrance into the brain by $\mathrm{M} 2$ and $\mathrm{M} 3$. M, molar.

incisors were noted on two of the affected animals (Figure $2 \mathrm{~b}$ and $2 \mathrm{~d}$ ), but not in the other six affected voles. When the surrounding tissues were removed, we noted overgrowth of all three molars on the apical side (Figure 3), but the protuberances were caused by overgrowth of the first molar (M1). The unrestricted continuous growth of the mandibular molars resulted in expansion of the mandibular bone, and the molars broke through the cortical bone in some cases. In one case, the
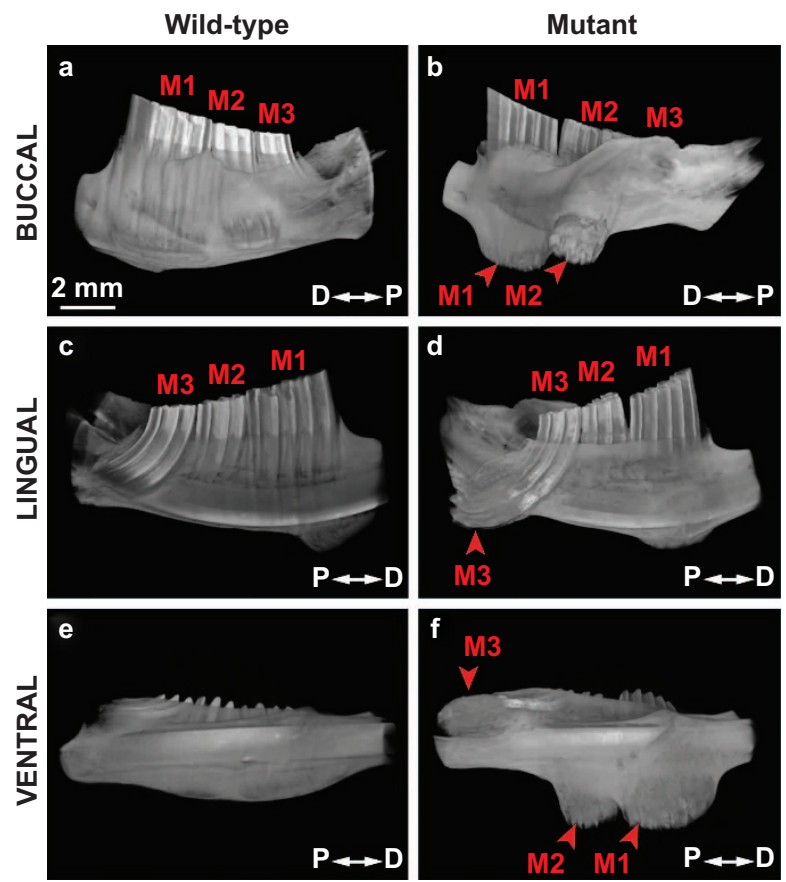

Figure 4 MicroCT analysis confirmed molar (M1, M2, M3) overgrowth in the mutant hemimandible as observed in the buccal, lingual and ventral views. D, distal; mciroCT, microcomputed tomography; P, proximal.

molar even broke through the skin, leading to infection and inflammation. Unchecked continuous growth also resulted in the maxillary molars breaking through the base of the cranial floor leading to invasion of the brain (Figure $3 \mathrm{e}-3 \mathrm{f}^{\prime}$ ). It is likely that maxillary molar invasion into the cranial vault influenced the health of the voles, and some of the affected voles appeared lethargic and ill. Analysis of the hemimandible by microcomputed tomography (microCT) confirmed the mandibular molar phenotype and also further revealed the extent to which the molars had overgrown (Figure 4). The first and second mandibular molars protruded from the buccal surface of the mandible though the cortical bone (Figure $4 \mathrm{~b}$ and $4 \mathrm{f}$ ), and the third molar (M3) was extended on the lingual side (Figure $4 \mathrm{~d}$ ).

We aimed to determine the mode of inheritance of the molar phenotype by mating mutants with other mutant or wild-type voles (Figure 5). We observed a complex, non-Mendelian, inheritance pattern over several generations, and the molar phenotype was no longer observed in the F2 generation.

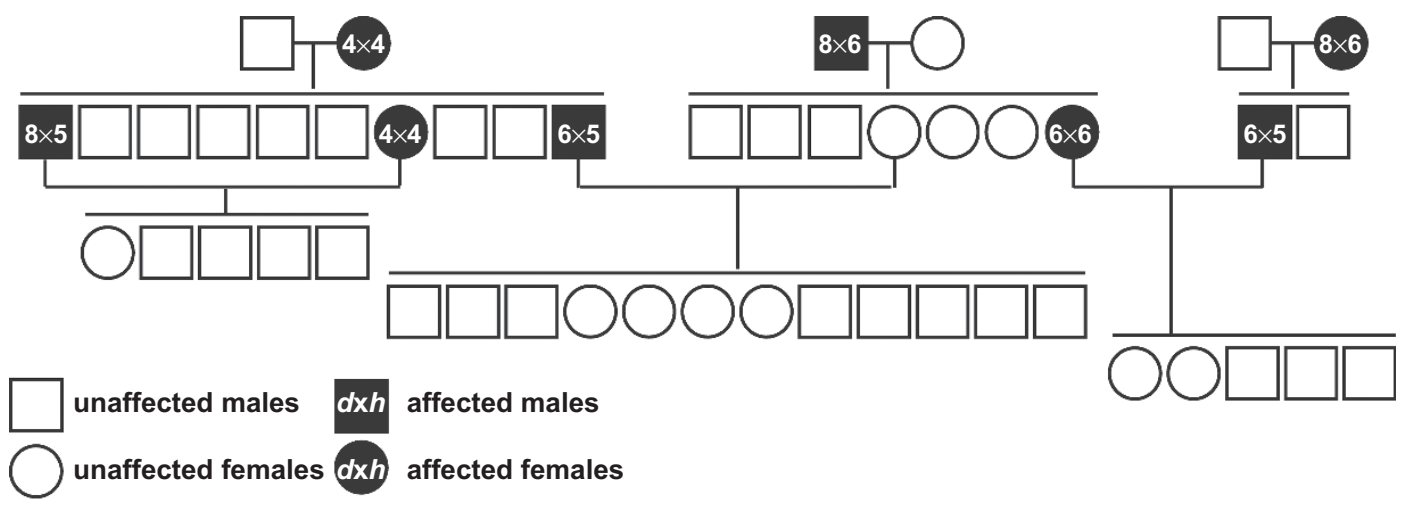

Figure 5 Family pedigree of mutant voles. The molar phenotype was lost after several generations of breeding. $d$, diameter ( $\mathrm{mm}) ; h$, height (mm). 


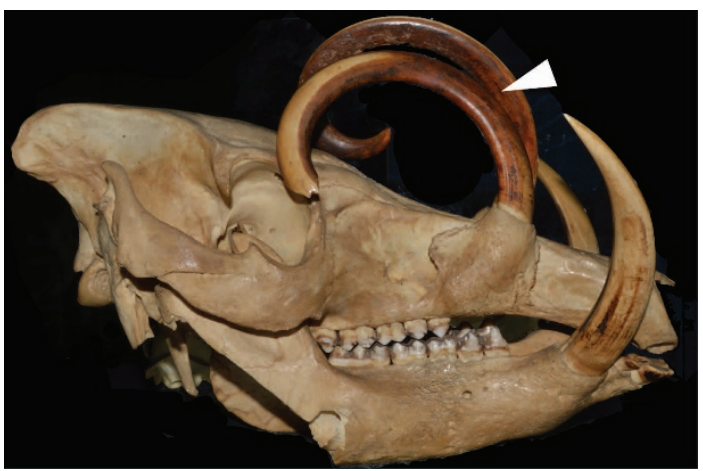

Figure 6 Skull of a Babirusa pig demonstrates the upper canine (white arrowhead) that grows dorsally out of the maxilla.

\section{DISCUSSION}

Here, we present a remarkable molar tooth phenotype in a colony of Prairie voles (Microtus ochrogaster) that appears to have resulted from a spontaneous mutation leading to the mis-regulation of continuously growing molars. Although stem cell-supplied growth is not as well characterized in rodent molars (compared to incisors), the similarities between mouse incisors and vole molars have previously been considered. ${ }^{7}$ Both incisors and molars are known to grow continuously in voles, but only the molars demonstrated an obvious defect in our mutants. The incisors of the affected animals, and particularly the labial CL located on the proximal incisor, appeared to be normal without any evidence of misregulated growth in six out of eight affected voles (data not shown). Possibly, the incisors in the two voles noted to have longer maxillary incisors (one is shown in Figure 2) were also misregulated. However, because incisors may become overgrown as a result of skeletal or dentoalveolar malocclusions resulting in the inability to properly gnaw down the incisor, this scenario seems unlikely. Therefore, our observations suggest that important gene regulatory differences exist between continuously growing incisors and molars in voles. Further analysis will be required to definitively determine whether gene regulatory differences exist.

Our attempts to understand the inheritance profile of the molar phenotype demonstrated complex, non-Mendelian ratios, and the molar phenotype was lost in the F2 generation (Figure 5). It is unclear why the molar phenotype was seemingly lost in the F2 generation. However, our study was somewhat complicated by difficulties in detecting the bilateral ventral protrusions by visual inspection or palpation, and the F2 molar phenotype may have been less severe. Additionally, the earliest time point that we detected the molar phenotype was 5 months of age. We housed the F2 voles for ca 8-12 months. Thus, the molar phenotype may arise later in the F2 generation. Despite these complications, our results suggest that the inheritance and severity of the molar phenotype are multigenic or multifactorial.

There are several possible reasons for molar-specific defects in the mutant voles. As mentioned above, the incisor and molar stem cell niches may not be as similar as previously hypothesized. In particular, the molars are required for mastication and receive the majority of the occlusal forces. The incisors are mainly used for pinching and tearing. Thus, one possibility is that the spontaneous mutation that led to abnormal molars in our vole colony did not affect the incisors perhaps by influencing components of a molar-specific mechanotransduction pathway. Second, there may be incisor- and molar-specific differences in the periodontal ligament anchorage of continuously growing teeth to the alveolar bone. Little is known about how continuously growing teeth are anchored to the bone. Thus, a molar-specific defect in anchorage could lead to uncontrolled apical molar growth. It is possible that the phenotypes reported here involving unchecked molar apical growth may have led to the evolution of tusks (similar to the male Babirusa pig) (Figure 6). In these animals, the upper canines grow dorsally passing through the maxilla to emerge and elongate (Figure 6). Interestingly, another distinct molar phenotype was reported in Pine voles (Microtus pinetorum), where the coronal portions of the molars became overgrown (rather than apical region overgrowth), and no incisor defects were noted. ${ }^{11}$ This finding suggests that mutations responsible for alterations in vole molars may be more common than previously thought.

In summary, we report several cases of voles with unrestricted molar growth that is likely due to the misregulation of dental stem cells arising from a spontaneous mutation. Further study of this molar phenotype may yield deeper insight into the regulation and evolution of continuously growing teeth.

\section{ACKNOWLEDGEMENTS}

This work was funded by the National Institutes of Health through grants R00DE022059 to Andrew H Jheon; DP2-OD007191 and R01-DE021420 to Ophir Klein; National Alliance for Research on Schizophrenia and Depression (NARSAD) grant to Devanand S Manoli; and DP1MH099900 to Nirao M Shah. The microCT imaging was performed by Sabra Djomehri at the Division of Biomaterials and Bioengineering MicroCT Imaging Facility at UCSF, which is supported by the Department of Health and Human Services/NIH S10 Shared Instrumentation Grant (S10RR026645) and the Departments of Preventive and Restorative Dental Sciences and Orofacial Sciences, School of Dentistry, UCSF. We would like to thank Dr Drew Noden (Cornell University, Ithaca, NY, USA) for informing us of the Babirusa pig and the California Academy of Sciences, San Francisco, CA, USA, for the skull specimen of the Babirusa pig (catalog number CAS MAM 22823).

1 Juuri E, Saito K, Ahtiainen L et al. Sox $2^{+}$stem cells contribute to all epithelial lineages of the tooth via Sfrp5 ${ }^{+}$progenitors. Dev Cell 2012; 23(2): 317-328.

2 Seidel K, Ahn CP, Lyons D et al. Hedgehog signaling regulates the generation of ameloblast progenitors in the continuously growing mouse incisor. Development 2010; 137(22): 3753-3761.

3 Biehs B, Hu JK, Strauli NB et al. BMI 1 represses Ink4a/Arf and Hox genes to regulate stem cells in the rodent incisor. Nat Cell Biol 2013; 15(7): 846-852.

4 Harada $\mathrm{H}$, Kettunen $\mathrm{P}$, Jung $\mathrm{HS}$ et al. Localization of putative stem cells in dental epithelium and their association with Notch and FGF signaling. J Cell Biol 1999; 147(1): 105-120.

5 Lapthanasupkul P, Feng J, Mantesso A et al. Ringla/b polycomb proteins regulate the mesenchymal stem cell niche in continuously growing incisors. Dev Biol 2012; 367(2): 140-153.

6 Zhao H, Feng J, Seidel $\mathrm{K}$ et al. Secretion of shh by a neurovascular bundle niche supports mesenchymal stem cell homeostasis in the adult mouse incisor. Cell Stem Cell 2014; 14(2): 160-173.

7 Goedendorp MM, van der Werf SP, Bleijenberg G et al. Does neuropsychological test performance predict outcome of cognitive behavior therapy for Chronic Fatigue Syndrome and what is the role of underperformance? J Psychosom Res 2013; 75(3): 242-248.

8 Harada $\mathrm{H}$, Toyono $\mathrm{T}$, Toyoshima $\mathrm{K}$ et al. FGF10 maintains stem cell compartment in developing mouse incisors. Development 2002; 129(6): 1533-1541.

9 United States Congress, Subtitle F. Animal welfare//United States Code congressional and administrative news: 99th Congress. St Paul: West Publishing, 1985: 2518-2524.

10 National Research Council, Institute of Laboratory Animal Research, Committee for the Update of the Guide for the Care and Use of Laboratory Animals. Guide for the care and use of laboratory animals. 8th ed. Washington: National Academies Press, 2011.

11 Harvey SB, Alworth LC, Blas-Machado U. Molar malocclusions in pine voles (Microtus pinetorum). J Am Assoc Lab Anim Sci 2009; 48(4): 412-415.

\section{(c) (i) $(-)$ This work is licensed under a Creative Commons Attribution-}

NonCommercial-NoDerivs 3.0 Unported License. The images or other third party material in this article are included in the article's Creative Commons license, unless indicated otherwise in the credit line; if the material is not included under the Creative Commons license, users will need to obtain permission from the license holder to reproduce the material. To view a copy of this license, visit http://creativecommons.org/licenses/ by-nc-nd/3.0/ 